\title{
Exact Solutions and Conserved Vectors of the Two-Dimensional Generalized Shallow Water Wave Equation
}

\author{
Chaudry Masood Khalique ${ }^{1,2, *,+}$ (D) and Karabo Plaatjie ${ }^{1, \dagger}$ \\ 1 International Institute for Symmetry Analysis and Mathematical Modelling, Department of Mathematical \\ Sciences, Mafikeng Campus, North-West University, Private Bag X 2046, Mmabatho 2735, South Africa; \\ 25451308@student.g.nwu.ac.za \\ 2 Department of Mathematics and Informatics, Azerbaijan University, Jeyhun Hajibeyli Str., 71, \\ Baku AZ1007, Azerbaijan \\ * Correspondence: Masood.Khalique@nwu.ac.za \\ + These authors contributed equally to this work.
}

check for updates

Citation: Khalique, C.M.; Plaatjie, K.

Exact Solutions and Conserved

Vectors of the Two-Dimensional Generalized Shallow Water Wave

Equation. Mathematics 2021, 9, 1439.

https://doi.org/10.3390/math9121439

Academic Editor: Andrei Dmitrievich Polyanin

Received: 26 March 2021

Accepted: 10 May 2021

Published: 21 June 2021

Publisher's Note: MDPI stays neutral with regard to jurisdictional claims in published maps and institutional affiliations.

Copyright: (c) 2021 by the authors. Licensee MDPI, Basel, Switzerland. This article is an open access article distributed under the terms and conditions of the Creative Commons Attribution (CC BY) license (https:// creativecommons.org/licenses/by/ $4.0 /)$.

\begin{abstract}
In this article, we investigate a two-dimensional generalized shallow water wave equation. Lie symmetries of the equation are computed first and then used to perform symmetry reductions. By utilizing the three translation symmetries of the equation, a fourth-order ordinary differential equation is obtained and solved in terms of an incomplete elliptic integral. Moreover, with the aid of Kudryashov's approach, more closed-form solutions are constructed. In addition, energy and linear momentum conservation laws for the underlying equation are computed by engaging the multiplier approach as well as Noether's theorem.
\end{abstract}

Keywords: two-dimensional generalized shallow water wave equation; Lie point symmetries; Kudryashov's method; conservation laws; Noether's theorem

\section{Introduction}

It is widely known that most physical phenomena of the natural world are governed by nonlinear partial differential equations (NPDEs). Such equations arise in several physical and other problems such as in the study of electromagnetism, electrodynamics, fluid flow, elasticity, propagation of heat or sound, quantum mechanics, meteorology, and oceanography, just to mention a few. Some of these models include the modified ZakharovKuznetsov model [1] that recounts the ion-acoustic drift solitary waves existing in a magnetoplasma with electron-positron ions, which are found in a primordial universe. A generalized system of three-dimensional modified Kadomtsev-Petviashvili-Burgerstype equation, which describes ion-acoustic and dust-magneto-acoustic waves in one of cosmic or laboratory dusty plasmas, was studied in [2]. Moreover, in [3], the vector bright solitons of the Fokas-Lenells system, which models the femtosecond pulses in an optical fibre, was investigated. In addition, the Boussinesq-Burgers-type system of equations, which delineates shallow water waves appearing close to lakes or ocean beaches, was studied in [4]. The list continues; see also [5-15].

To obtain a good understanding of physical phenomena that are governed by NPDEs, it is imperative to look for their closed-form solutions. However, this is an arduous exercise, and for several years, researchers and scientists have been working on developing methods for finding closed-form solutions.

A good number of methods for obtaining closed-form solutions of NPDEs have been proposed. We have, amongst others, the following various special methods for finding closed-form solutions of NPDEs: the inverse scattering transform technique [16], the bifurcation method [17], the simplest equation technique [18], the extended simplest equation technique [19], Kudryashov's technique [20], Hirota's technique [21], Bäcklund transformation [22], Darboux transformation [23], the homogeneous balance method [24], $\left(G^{\prime} / G\right)$-expansion technique [25], Lie symmetry method [26-28], etc. 
It was during the halfway point of the nineteenth century that Sophus Lie, a Norwegian mathematician, endowed the study of continuous transformation groups, presently called Lie groups. These groups have had an intense impact on pure and applied mathematics in addition to engineering, physics, and other applied sciences. It provides the most effective and powerful techniques for obtaining closed-form solutions to NPDEs. For example, see [26-33]. It is worth noting here that the notion of a Galois group had influenced Lie's work on differential equations (DEs).

In the study of DEs, conservation laws play a crucial part. Conservation laws can be used to check whether a given differential equation is integrable. The accuracy of numerical solution methods can be checked by invoking conservation laws. They can be utilized in constructing solutions of partial differential equations (PDEs) by reducing their order. Conservation laws refer to certain physical properties that do not change subsequently within an isolated physical system. For example, conservation of energy, linear momentum, angular momentum, and electric charge. Recently, exact solutions were obtained for some PDEs using conservation laws. For differential equations that arise from the variational principle, there exists a connection between the symmetries of differential equations and conservation laws. This fact was established by Emmy Noether, a German mathematician, in 1918 and is stated in Noether's theorem. For more details, see for example [34-45] and the references therein.

In this work, we study the generalized two-dimensional generalized shallow water wave equation, given by [46]

$$
u_{t x}+\alpha u_{y} u_{x x}+2 \alpha u_{x} u_{x y}+\beta u_{x y}+v u_{x x x y}=0,
$$

where $\alpha, \beta$, and $v$ are nonzero constants. Here, $t, x$, and $y$ represent, respectively, time and two scaled space coordinates whereas $u(t, x, y)$ represents the height of the Riemann wave.

In [46], the authors presented painlévé analysis, nonlocal symmetries, and exact solutions of a variable coefficients form of (1), whereas in [47], the binary Bell polynomials technique was employed to a variable coefficient form of Equation (1). We note that, in the study of fluids, this equation decomposes to well-known equations when taking special cases of constants $\alpha, \beta$, and $v$. For example, when $\alpha=-2, \beta=0$, and $v=-1$, it becomes a $(2+1)$-dimensional breaking soliton [48]:

$$
u_{t x}-2 u_{y} u_{x x}-4 u_{x} u_{x y}-u_{x x x y}=0,
$$

which is one of the models used in describing the two-dimensional interplay of a Riemann wave distributed along the $y$-axis with a long wave generated along the $x$-axis. Wazwaz [49] obtained solutions of this equation using the modified form of Hirota's bilinear method. The authors in [48] presented solutions of Equation (2) using the symmetry method. In the case where $\alpha=2, \beta=0$, and $v=1$, Equation (1) transforms to a $(2+1)$-dimensional Calogero-Bogoyavlenskii-Schiff [50]

$$
u_{t x}+2 u_{y} u_{x x}+4 u_{x} u_{x y}+u_{x x x y}=0
$$

which describes the exchange of a long generating wave in the $x$ direction with a Riemann generating wave in the $y$ direction. Moreover, the researcher indicated that Equation (3) possesses soliton as well as N-soliton solutions, which are smooth in one coordinate. In [51], Wazwaz invoked Hirota's bilinear approach to secure solutions of (3) and further utilized the tanh-coth method to gain its travelling wave solutions in [52]. The authors in [53] derived a Bäcklund transformation alongside a Lax pair of the form in the CalogeroBogoyavlenskii-Schiff Equation (3) via singular manifold method. Furthermore, in [54], the authors invoked a tanh function as well as improved the $\left(G^{\prime} / G\right)$ - expansion methods to construct travelling wave solutions of (3). It was also revealed that some analytic solutions secured via these methods are analogous. However, a generalized version of (3), namely

$$
u_{t x}+a u_{x} u_{x y}+b u_{y} u_{x x}+u_{x x x y}=0
$$


has been studied by many researchers by simply replacing 4 by $a$ and 2 by $b$ in (3). For instance, the $\left(G^{\prime} / G\right)$ - expansion technique was invoked to achieve some travelling wave solutions of (4) in [55]. Moreover, in [56], the authors obtained breather-type and analytic soliton solutions of (4) by utilizing Hirota's bilinear method and the extended homoclinic test technique. Using the idea of a tanh-coth approach, some closed-form solutions of (4) were obtained in [57]. Furthermore, sine-cosine and complex techniques were engaged to secure, respectively, analytic and meromorphic exact solutions of (4) in [58,59].

The formation of this paper is as follows: In Section 2, first, we employ Lie's theory to reduce (1) to an ordinary differential equation (ODE). Closed-form solutions are then derived by an integration of the ODE and by invoking Kudryashov's method. Thereafter, in Section 3, we construct conserved densities and spatial fluxes for (1) by invoking the multiplier method and Noether's theorem. Finally, we present concluding remarks in Section 4 .

\section{Exact Solutions}

In this section, we derive exact solutions of the two-dimensional generalized shallow water wave Equation (1). We begin by deriving symmetries of (1) and thereafter use them to obtain an ODE. We then employ the direct integration procedure together with Kudryashov's method to construct closed-form solutions of (1).

\subsection{Symmetries and Symmetry Reductions}

Consider the symmetry group of (1) brought about by

$$
U=T \frac{\partial}{\partial t}+X \frac{\partial}{\partial x}+Y \frac{\partial}{\partial y}+\eta \frac{\partial}{\partial u},
$$

where all $T, X, Y$, and $\eta$ are functions of variables $t, x, y$, and $u$. Using the fourth prolongation $\mathrm{pr}^{(4)} U$ in (1) and with the help of Maple, we obtain an overdetermined system of linear homogeneous PDEs:

$$
\begin{aligned}
& T_{u}=0, T_{x}=0, T_{y}=0, X_{y}=0, Y_{u}=0, Y_{x}=0, Y_{t t}=0, T_{t t t}=0, X_{u}=0, Y_{y y}=0, \\
& 2 \eta_{u}+T_{t}-Y_{y}=0,2 \alpha \eta_{x}+\beta T_{t}-\beta Y_{y}-Y_{t}=0, \alpha \eta_{y}+X_{t}=0,2 Y_{t y}-T_{t t}=0, \\
& 2 X_{x}-T_{t}+Y_{y}=0 .
\end{aligned}
$$

The above linear PDEs may be easily solved and yield the following eight Lie point symmetries:

$$
\begin{aligned}
& U_{1}=\frac{\partial}{\partial t^{\prime}}, U_{2}=\frac{\partial}{\partial x}, U_{3}=\frac{\partial}{\partial y}, U_{4}=\frac{\partial}{\partial u}, \\
& U_{5}=2 \alpha t \frac{\partial}{\partial y}+x \frac{\partial}{\partial u}, U_{6}=2 \alpha t \frac{\partial}{\partial t}+\alpha x \frac{\partial}{\partial x}-(\beta x+\alpha u) \frac{\partial}{\partial u}, \\
& U_{7}=\alpha x \frac{\partial}{\partial x}-2 \alpha y \frac{\partial}{\partial y}-(\alpha u+\beta x) \frac{\partial}{\partial u^{\prime}} \\
& U_{8}=2 \alpha t^{2} \frac{\partial}{\partial t}+\alpha t x \frac{\partial}{\partial x}+2 \alpha t y \frac{\partial}{\partial y}-(\beta t x-x y+\alpha t u) \frac{\partial}{\partial u} .
\end{aligned}
$$

Consider the symmetry $U=U_{1}+a U_{2}+b U_{3}$, where $a$ and $b$ are constants. Using this symmetry $U$, we reduce (1) to a PDE with one less independent variable, that is, two independent variables. The symmetry $U$ gives three invariants:

$$
f=x-a t, g=y-b t, \theta=u .
$$


Using these invariants, Equation (1) reduces to

$$
v \theta_{f f f g}+\beta \theta_{f g}+\alpha \theta_{g} \theta_{f f}+2 \alpha \theta_{f} \theta_{f g}-b \theta_{f g}-a \theta_{f f}=0
$$

which has

$$
\begin{aligned}
& R_{1}=\frac{\partial}{\partial f}, R_{2}=\frac{\partial}{\partial g}, R_{3}=\alpha H(g) \frac{\partial}{\partial g}+a H(g) \frac{\partial}{\partial \theta} \\
& R_{4}=\alpha f \frac{\partial}{\partial f}-\alpha g \frac{\partial}{\partial g}+(b f-\beta f-\alpha \theta) \frac{\partial}{\partial \theta}
\end{aligned}
$$

as its symmetries. The symmetry $R=R_{1}+c R_{2}$, with $c$ a constant, provides two invariants $q=g-c f$ and $\mathcal{U}=\theta$. These invariants transform (7) into a nonlinear ordinary differential equation (NLODE)

$$
k_{1} \mathcal{U}^{\prime \prime \prime \prime}(q)-k_{2} \mathcal{U}^{\prime}(q) \mathcal{U}^{\prime \prime}(q)+k_{3} \mathcal{U}^{\prime \prime}(q)=0
$$

where $k_{1}=b-a c^{2}-\beta, k_{2}=-3 \alpha c, k_{3}=-v c^{2}$ and $q=(a c-b) t-c x+y$.

\subsection{Solution Using Direct Integration}

In this subsection, we derive a solution of the two-dimensional generalized shallow water Equation (1) by direct integration of the ODE (8). Taking $\mathcal{U}^{\prime}(q)=\mathcal{V}(q)$, Equation (8) becomes

$$
k_{1} \mathcal{V}^{\prime \prime \prime}-k_{2} \mathcal{V} \mathcal{V}^{\prime}+k_{3} \mathcal{V}^{\prime}=0
$$

Twice integration of (9) with respect to $q$ gives

$$
\frac{1}{2} k_{1} \mathcal{V}^{\prime 2}-\frac{1}{6} k_{2} \mathcal{V}^{3}+\frac{1}{2} k_{3} \mathcal{V}^{2}+r_{1} \mathcal{V}+r_{2}=0
$$

with $r_{1}$ and $r_{2}$ constants. Equation (10) can be rewritten as

$$
\mathcal{V}^{\prime 2}=\frac{k_{2}}{3 k_{1}} \mathcal{V}^{3}-\frac{k_{3}}{k_{1}} \mathcal{V}^{2}-\frac{2 r_{1}}{k_{1}} \mathcal{V}-\frac{2 r_{2}}{k_{1}}
$$

Assume that $n_{1}, n_{2}$, and $n_{3}$ are roots of

$$
\mathcal{V}^{3}-\frac{3 k_{3}}{k_{2}} \mathcal{V}^{2}-\frac{6 r_{1}}{k_{2}} \mathcal{V}-\frac{6 r_{2}}{k_{2}}=0
$$

with $n_{1}>n_{2}>n_{3}$. Equation (11) now becomes

$$
\mathcal{V}^{\prime 2}=\frac{k_{2}}{3 k_{1}}\left(\mathcal{V}-n_{1}\right)\left(\mathcal{V}-n_{2}\right)\left(\mathcal{V}-n_{3}\right)
$$

and its solution could be written in terms of the Jacobi elliptic function [60-62]

$$
\mathcal{V}(q)=n_{2}+\left(n_{1}-n_{2}\right) \mathrm{cn}^{2}\left\{\sqrt{\frac{k_{2}\left(n_{1}-n_{3}\right)}{12 k_{1}}} q, R^{2}\right\}, R^{2}=\frac{n_{1}-n_{2}}{n_{1}-n_{3}}
$$

where $\mathrm{cn}$ is the elliptic cosine function. Integrating Equation (12) with respect to $q$ and returning to the original variables, we accomplish the solution of (1) as 


$$
\begin{aligned}
u(t, x, y)= & \sqrt{\frac{12 k_{1}\left(n_{1}-n_{2}\right)^{2}}{k_{2}\left(n_{1}-n_{3}\right) R^{8}}}\left\{\text { EllipticE }\left[\operatorname{sn}\left(\sqrt{\frac{k_{2}\left(n_{1}-n_{3}\right)}{12 k_{1}}} q, R^{2}\right), R^{2}\right]\right\} \\
& +\left\{n_{2}-\left(n_{1}-n_{2}\right) \frac{1-R^{4}}{R^{4}}\right\} q+K
\end{aligned}
$$

with $q=(a c-b) t-c x+y, K$ being a constant, and EllipticE $[g, k]$ being the incomplete elliptic integral given as [60]

$$
\text { EllipticE }[g, k]=\int_{0}^{g} \sqrt{\frac{1-k^{2} s^{2}}{1-s^{2}}} d s .
$$

The wave profile of the periodic solution (13) for parametric values $k_{1}=70$, $k_{2}=10, a=-4, b=0.2, c=0.6, K=1, n_{1}=100, n_{2}=50.05$, and $n_{3}=-60$ at $t=-14$, can be seen in Figure 1 .
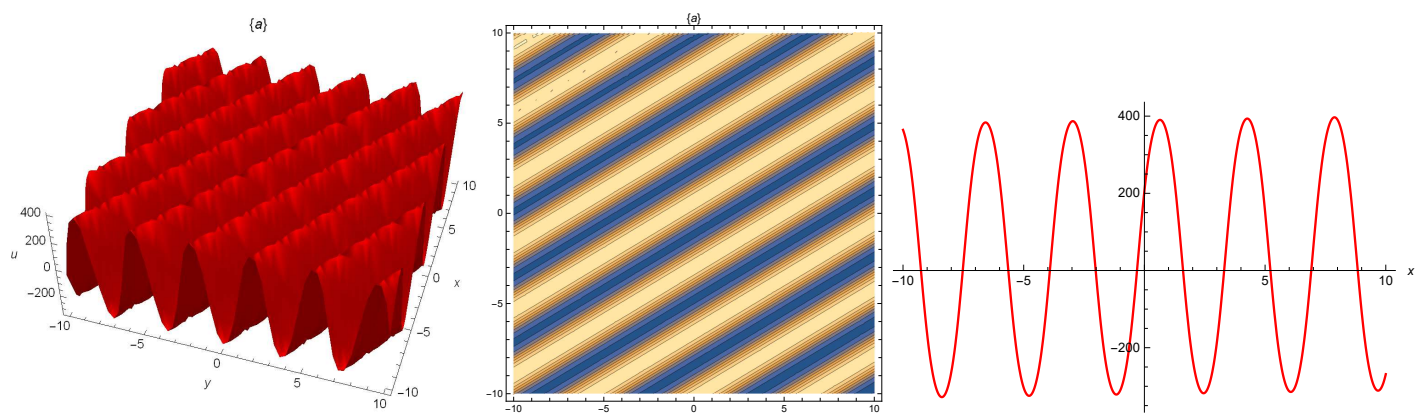

Figure 1. The 3D and 2D solution profiles of (13).

\subsection{Solution via Kudryashov's Method}

In this subsection, we present the solution of (1), which is obtained by employing Kudryashov's method [20]. This method is one of the most popular approaches for determining closed-form solutions of NPDEs. The first step is to reduce the NPDE (1) to a nonlinear ODE, which we already performed using the Lie symmetries in the previous section. Thus, we work with the ODE (8). We suppose that a solution of (8) can be expressed as

$$
\mathcal{U}(q)=\sum_{n=0}^{N} A_{n} Y^{n}(q)
$$

where $Y(q)$ satisfies the first-order nonlinear ODE

$$
Y^{\prime}(q)=Y^{2}(q)-Y(q) .
$$

We note that the solution of (16) is

$$
Y(q)=\frac{1}{1+\exp q} .
$$


For Equation (8), the balancing procedure yields $N=1$. Thus, from (15), we have

$$
\mathcal{U}(q)=A_{0}+A_{1} Y(q)
$$

Now substituting (18) into (8) and using (16), we obtain

$$
\begin{aligned}
& 2 b A_{1} c Y^{3}(q)-2 a A_{1} c^{2} Y^{3}(q)+3 a A_{1} c^{2} Y^{2}(q)-3 b A_{1} c Y^{2}(q) \\
& -A_{1} c^{2} Y(q) a+b A_{1} c Y(q)+6 \alpha A_{1}^{2} c^{2} Y^{5}(q)-15 \alpha A_{1}{ }^{2} c^{2} Y^{4}(q) \\
& +12 \alpha A_{1}^{2} c^{2} Y^{3}(q)-3 \alpha A_{1}^{2} c^{2} Y^{2}(q)-2 \beta A_{1} c Y^{3}(q)+3 \beta A_{1} c Y^{2}(q) \\
& -\beta A_{1} c Y(q)-24 v A_{1} c^{3} Y^{5}(q)+60 v A_{1} c^{3} Y^{4}(q)-50 v A_{1} c^{3} Y^{3}(q) \\
& +15 v A_{1} c^{3} Y^{2}(q)-v A_{1} c^{3} Y(q)=0 .
\end{aligned}
$$

Separating on the powers of $Y(q)$ gives the algebraic equations for the coefficients $A_{0}$ and $A_{1}$ as

$$
\begin{aligned}
& \alpha A_{1}^{2} c^{2}-4 v A_{1} c^{3}=0, \\
& b c A_{1}-v A_{1} c^{3}-a c^{2} A_{1}-\beta A_{1} c=0, \\
& \beta A_{1} c-\alpha A_{1}^{2} c^{2}+5 v A_{1} c^{3}+a c^{2} A_{1}-b c A_{1}=0, \\
& 6 \alpha A_{1}^{2} c^{2}-25 v A_{1} c^{3}-a c^{2} A_{1}+b c A_{1}-\beta A_{1} c=0 .
\end{aligned}
$$

The solution of these equations is

$$
A_{0}=A_{0}, A_{1}=\frac{4 v c}{\alpha}, a=\frac{b-\beta-c^{2} v}{c} .
$$

Thus, the solution of (1) reads

$$
u(t, x, y)=A_{0}+\frac{4 v c}{\alpha\left(1+\exp \left\{\left(-\beta-c^{2} v\right) t-c x+y\right\}\right)}
$$

The wave profile of the solution (19) for parametric values $A_{0}=0, \alpha=1, \beta=0.05$, $c=0.01, v=1, t=0$ is presented in Figure 2.
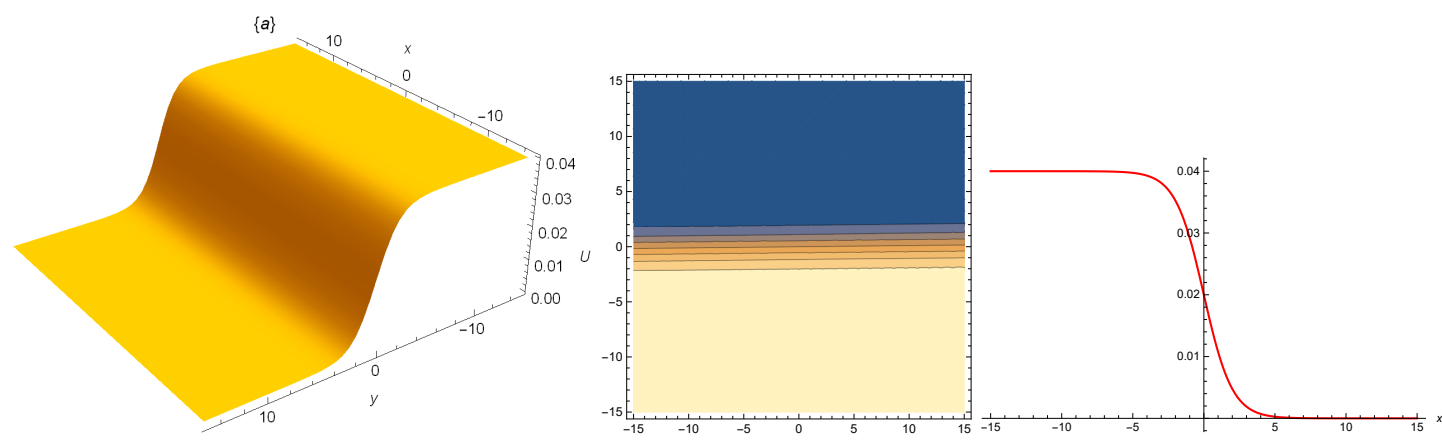

Figure 2. The 3D and 2D solution profile of (19).

Invariant solution using $U_{5}$

The Lie point symmetry $U_{5}$ produces the invariants $I_{1}=t, I_{2}=x$, and $I_{3}=u-$ $x y /(2 \alpha t)$. The use of these invariants furnishes us with the group invariant solution of (1) given by 


$$
u(t, x, y)=\frac{x y}{2 \alpha t}-\frac{\beta}{2 \alpha} x-\frac{C}{x}+h(t),
$$

where $C$ is a constant and $h$ is a function of $t$.

Invariant solution using $U_{6}$

The point symmetry $U_{6}$ of (1) yields the group invariant solution:

$$
u(t, x, y)=\frac{1}{\sqrt{t}} G(f, g)-\frac{\beta}{2 \alpha} x, f=\frac{x}{\sqrt{t}}, g=y .
$$

The substitution of this expression for $u$ into (1) gives the PDE

$$
2 \nu G_{f f f g}+4 \alpha G_{f} G_{f g}+2 \alpha G_{g} G_{f f}-f G_{f f}-2 G_{f}=0,
$$

for which the symmetries are

$$
\Gamma_{1}=\frac{\partial}{\partial g}, \Gamma_{2}=\frac{\partial}{\partial G}, \Gamma_{3}=2 \alpha \frac{\partial}{\partial f}+g \frac{\partial}{\partial g}, \Gamma_{4}=f \frac{\partial}{\partial f}-2 g \frac{\partial}{\partial g}-G \frac{\partial}{\partial G} .
$$

The symmetry $\Gamma_{3}$ gives the invariant solution $G(f, g)=U(z)+f g /(2 \alpha)$, where $z=g$. This expression for $G$ satisfies the Equation (22) for arbitrary function $U$. Thus, the group-invariant solution under $U_{6}$ of $(1)$ is

$$
u(t, x, y)=\frac{x y}{2 \alpha t}-\frac{\beta}{2 \alpha} x-\frac{U(y)}{\sqrt{t}},
$$

where $U(y)$ is an arbitrary function of $y$.

Invariant solution using $U_{7}$

Here, we use the symmetry operator $U_{7}$ to obtain the invariant solution associated with this symmetry. The usual procedure gives

$$
u(t, x, y)=\frac{1}{x} U(\xi)-\frac{\beta}{2 \alpha} x, \quad \xi=x^{2} y
$$

Substituting this expression of $u$ into Equation (1) gives the nonlinear ODE

$$
\alpha U^{\prime 2}+6 \alpha \xi U^{\prime} U^{\prime \prime}-2 \alpha U U^{\prime \prime}+3 v U^{\prime \prime}+4 v \xi^{2} U^{\prime \prime \prime \prime}+12 v \xi U^{\prime \prime \prime}=0 .
$$

Invariant solution using $U_{8}$

Finally, the use of symmetry $U_{8}$ gives the invariant solution

$$
u(t, x, y)=\frac{1}{\sqrt{t}} \Phi(f, g)+\frac{x y}{2 \alpha t}-\frac{\beta x}{2 \alpha},
$$

with $f=x / t^{1 / 2}$ and $g=y / t$, which when substituted in (1) gives the reduced PDE

$$
\alpha \Phi_{f f} \Phi_{g}+2 \alpha \Phi_{f} \Phi_{f g}+v \phi_{f f f g}=0
$$


The above PDE (28) has three Lie symmetries

$$
\Gamma_{1}=\frac{\partial}{\partial f}+F_{1}(g) \frac{\partial}{\partial g}, \Gamma_{2}=F_{2}(g) \frac{\partial}{\partial g}+\frac{\partial}{\partial \Phi}, \Gamma_{3}=f \frac{\partial}{\partial f}+F_{3}(g) \frac{\partial}{\partial g}-\Phi \frac{\partial}{\partial \Phi} .
$$

Now utilizing $\Gamma_{1}$ with $F_{1}(g)=1$, we perform a reduction of Equation (28). We obtain the invariant solution $\Phi(f, g)=U(z)$, where $z=g-f$. This reduces Equation (28) to an ODE

$$
v U^{\prime \prime \prime \prime}-3 \alpha U^{\prime} U^{\prime \prime}=0
$$

for which the solution is

$$
U(z)=-\sqrt{\frac{2^{8 / 3} v}{\alpha^{4 / 3}}} \zeta\left\{\sqrt{\frac{\alpha^{2 / 3}}{2^{4 / 3} v}} z+C_{0} ; s_{1}, s_{2}\right\}, s_{1}=\frac{2^{5 / 2}}{\sqrt[3]{\alpha}} C_{0}, s_{2}=2 C_{1},
$$

where $C_{0}, C_{1}$ are arbitrary constants and $\zeta$ is the Weierstrass zeta function [60]. Thus, the solution of (1) is

$$
u(t, x, y)=-\sqrt{\frac{2^{8 / 3} v}{\alpha^{4 / 3} t}} \zeta\left\{\sqrt{\frac{\alpha^{2 / 3}}{2^{4 / 3} v}}\left(\frac{y}{t}-\frac{x}{\sqrt{t}}\right)+C_{0} ; s_{1}, s_{2}\right\}+\frac{x y}{2 \alpha t}-\frac{\beta x}{2 \alpha} .
$$

For the operator $\Gamma_{2}$ with $F_{2}(g)=1$, we obtain the invariant solution $\Phi(f, g)=$ $U(f)+g$, which reduces (28) to the ODE $U_{f f}=0$, for which the solution is $U=C_{1} f+C_{2}$, where $C_{1}, C_{2}$ are constants. Reverting to the original variables, we attain the solution of (1) given by

$$
u(t, x, y)=\frac{C_{1} x}{t}+\frac{C_{2}}{\sqrt{t}}+\frac{y}{t^{3 / 2}}+\frac{x y}{2 \alpha t}-\frac{\beta x}{2 \alpha} .
$$

The dynamic behaviour of the solution (32), for parametric values $\alpha=10, \beta=5$, $C_{1}=0.3, C_{2}=0.01$, and $x=0.5$, is sketched in Figure 3 .

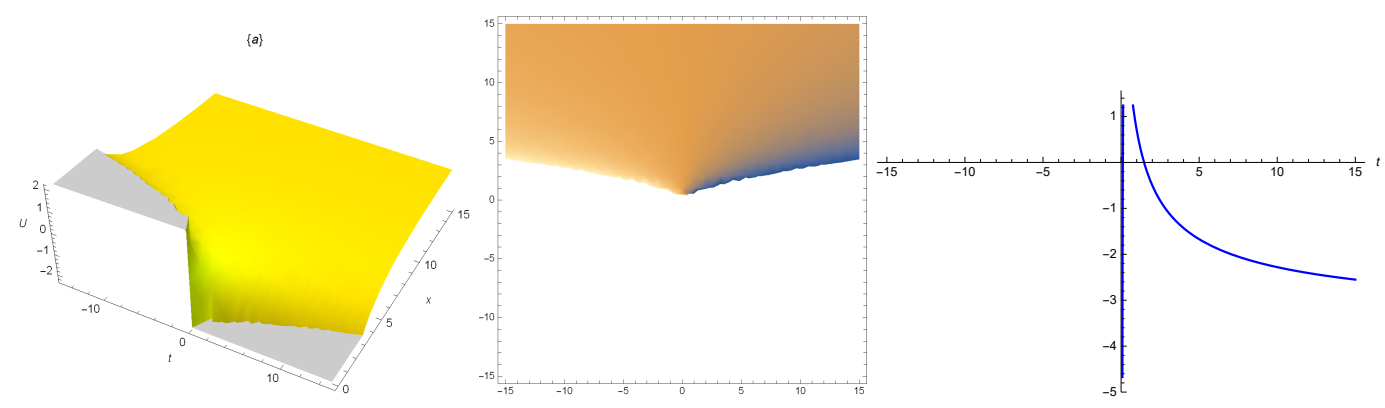

Figure 3. The 3D and 2D solution profiles of (32).

\section{Conservation Laws}

We now construct conservation laws for the two-dimensional generalized constantcoefficient shallow water Equation (1). To achieve this task, we utilize two methods; the first one is the multiplier method, and the second is classical Noether's theorem. 


\subsection{Conservation Laws Utilizing the Multiplier Method}

We determine the conserved vectors of Equation (1) by employing the multiplier method [26]. Here, we seek first-order multipliers $\mathcal{Q}=\mathcal{Q}\left(t, x, y, u, u_{t}, u_{x}, u_{y}\right)$, which are established from

$$
\frac{\delta}{\delta u}\left[\mathcal{Q}\left(u_{t x}+\alpha u_{y} u_{x x}+2 \alpha u_{x} u_{x y}+\beta u_{x y}+v u_{x x x y}\right)\right]=0,
$$

where $\delta / \delta u$ is the Euler operator [37]

$$
\frac{\delta}{\delta u}=\frac{\partial}{\partial u}+\sum_{s=1}^{\infty}(-1)^{s} D_{i_{1}} \cdots D_{i_{s}} \frac{\partial}{\partial u_{i_{1} \cdots i_{s}}}
$$

and for our purpose

$$
\frac{\delta}{\delta u}=\partial_{u}-D_{x} \partial_{u_{x}}-D_{y} \partial_{u_{y}}+D_{t} D_{x} \partial_{u_{t x}}+D_{x}^{2} \partial_{u_{x x}}+D_{x} D_{y} \partial_{u_{x y}}+D_{x}^{3} D_{y} \partial_{u_{x x x y}}
$$

with $D_{t}, D_{x}$, and $D_{y}$ being the total derivative operators given by

$$
\begin{aligned}
& D_{t}=\partial_{t}+u_{t} \partial_{u}+u_{t t} \partial_{u_{t}}+u_{t x} \partial_{u_{x}}+u_{t y} \partial_{u_{y}}+\cdots \\
& D_{x}=\partial_{x}+u_{x} \partial_{u}+u_{t x} \partial_{u_{t}}+u_{x x} \partial_{u_{x}}+u_{x y} \partial_{u_{y}}+\cdots \\
& D_{y}=\partial_{y}+u_{y} \partial_{u}+u_{t y} \partial_{u_{t}}+u_{x y} \partial_{u_{x}}+u_{y y} \partial_{u_{y}}+\cdots
\end{aligned}
$$

Expanding (33) and separating on the appropriate derivatives of $u$, we obtain twentysix multiplier-determining equations:

$$
\begin{aligned}
& \mathcal{Q}_{t x}+\alpha u_{x} \mathcal{Q}_{x y}+\beta \mathcal{Q}_{x y}=0, \mathcal{Q}_{t u}+\alpha \mathcal{Q}_{x y}=0, \mathcal{Q}_{t u_{t}}+4 \mathcal{Q}_{u}=0, \mathcal{Q}_{x u_{t}}=0, \mathcal{Q}_{x y}=0, \\
& \mathcal{Q}_{x u_{x}}-\mathcal{Q}_{u}=0, \mathcal{Q}_{t u_{x}}-2 \alpha u_{y} \mathcal{Q}_{u}+\alpha \mathcal{Q}_{y}=0, \mathcal{Q}_{t u_{y}}-2 \alpha u_{x} \mathcal{Q}_{u}+2 \alpha \mathcal{Q}_{x}-2 \beta \mathcal{Q}_{u}=0, \\
& \mathcal{Q}_{x x}=0, \mathcal{Q}_{u x}=0, \mathcal{Q}_{y y}=0, \mathcal{Q}_{u y}=0, \mathcal{Q}_{y u_{t}}=0, \mathcal{Q}_{y u_{x}}=0, \mathcal{Q}_{y u_{y}}-2 \mathcal{Q}_{u}=0, \\
& \mathcal{Q}_{u u}=0, \mathcal{Q}_{u u_{t}}=0, \mathcal{Q}_{u u_{x}}=0, \mathcal{Q}_{u u_{y}}=0, \mathcal{Q}_{u_{t} u_{t}}=0, \mathcal{Q}_{u_{t} u_{x}}=0, \mathcal{Q}_{u_{t} u_{y}}=0, \\
& \mathcal{Q}_{u_{x} u_{x}}=0, \mathcal{Q}_{u_{x} u_{y}}=0, \mathcal{Q}_{u_{y} u_{y}}=0, \mathcal{Q}_{x u_{y}}=0,
\end{aligned}
$$

for which the solution is

$$
\begin{aligned}
\mathcal{Q}= & y F^{\prime}(t)+G(t)-\alpha u_{x} F(t)-2 K_{5} \alpha t^{2} u_{t}-K_{5} \alpha t x u_{x}-2 K_{5} \alpha t y u_{y} \\
& -K_{5} \alpha t u+2 K_{6} \beta t u_{y}-2 K_{1} \alpha t u_{y}-K_{5} \beta t x+4 K_{6} t u_{t}+K_{6} x u_{x} \\
& +2 K_{6} y u_{y}+K_{5} x y+K_{6} u+K_{2} u_{t}+K_{3} u_{x}+K_{4} u_{y}+K_{1} x
\end{aligned}
$$

where $F$ and $G$ are arbitrary functions of $t$ and $K_{i}$, where $i=1, \ldots, 6$, are constants of integration. The conserved quantities of Equation (1) are constructed using the divergence identity

$$
D_{t} T^{t}+D_{x} T^{x}+D_{y} T^{y}=\mathcal{Q}\left(u_{t x}+\alpha u_{y} u_{x x}+2 \alpha u_{x} u_{x y}+\beta u_{x y}+v u_{x x x y}\right),
$$

where $T^{t}$ represents the conserved density and $T^{x}$ and $T^{y}$ are spatial fluxes. Thus, after some reckoning, we obtain low-order conservation laws corresponding to the eight multipliers and these are given below. 
Case 1. For $\mathcal{Q}_{1}=x-2 \alpha t u_{y}$, we have the conserved vector

$$
\begin{aligned}
T_{1}^{t}= & \frac{1}{2} \alpha t u u_{x y}-\frac{1}{2} \alpha t u_{x} u_{y}+\frac{1}{2} x u_{x}-\frac{1}{2} u \\
T_{1}^{x}= & \frac{2}{3} \alpha^{2} t u u_{x} u_{y y}+\frac{2}{3} \alpha^{2} t u u_{x y} u_{y}-\frac{4}{3} \alpha^{2} t u_{x} u_{y}^{2}+\frac{3}{4} \alpha v t u u_{x x y y}+\frac{1}{2} \alpha \beta t u u_{y y}-\frac{1}{2} \alpha v t u_{x} u_{x y y} \\
& +\frac{1}{4} \alpha v t u_{x x} u_{y y}-\alpha v t u_{x x y} u_{y}+\frac{1}{2} \alpha v t u_{x y}{ }^{2}-\frac{1}{2} \alpha \beta t u_{y}^{2}+\frac{1}{2} \alpha t u u_{t y}-\frac{1}{2} \alpha t u_{t} u_{y}+\alpha x u_{x} u_{y} \\
& +\frac{1}{2} \beta x u_{y}+\frac{3}{4} v x u_{x x y}-\frac{1}{2} v u_{x y}+\frac{1}{2} x u_{t}, \\
T_{1}^{y}= & -\frac{2}{3} \alpha^{2} t u u_{x} u_{x y}-\frac{2}{3} \alpha^{2} t u u_{x x} u_{y}-\frac{2}{3} \alpha^{2} t u_{x}{ }^{2} u_{y}-\frac{3}{4} \alpha v t u u_{x x x y}-\frac{1}{2} \alpha \beta t u u_{x y}-\frac{1}{4} \alpha v t u_{x} u_{x x y} \\
& -\frac{1}{2} \alpha \beta t u_{x} u_{y}+\frac{1}{4} \alpha v t u_{x x} u_{x y}-\frac{1}{4} \alpha v t u_{x x x} u_{y}-\alpha t u u_{t x}+\frac{1}{2} \alpha x u_{x}{ }^{2}-\frac{1}{2} \alpha u u_{x}+\frac{1}{2} \beta x u_{x} \\
& -\frac{1}{2} \beta u+\frac{1}{4} v x u_{x x x}-\frac{1}{4} v u_{x x} ;
\end{aligned}
$$

Case 2. For $\mathcal{Q}_{2}=u_{t}$, we have the conserved vector

$$
\begin{aligned}
T_{2}^{t}= & \frac{2}{3} \alpha u u_{x} u_{x y}+\frac{1}{3} \alpha u u_{x x} u_{y}+\frac{1}{2} v u u_{x x x y}+\frac{1}{2} \beta u u_{x y}+\frac{1}{4} u u_{t x}+\frac{1}{4} u_{t} u_{x}, \\
T_{2}^{x}= & -\frac{3}{8} v u u_{t x x y}+\frac{3}{8} v u_{t} u_{x x y}-\frac{1}{4} v u_{t x} u_{x y}+\frac{1}{8} v u_{t x x} u_{y}+\frac{1}{4} v u_{t x y} u_{x}-\frac{1}{8} v u_{t y} u_{x x}-\frac{1}{3} \alpha u u_{t x} u_{y} \\
& -\frac{1}{3} \alpha u u_{t y} u_{x}-\frac{1}{4} \beta u u_{t y}-\frac{1}{4} u u_{t t}+\frac{2}{3} \alpha u_{t} u_{y} u_{x}+\frac{1}{4} \beta u_{t} u_{y}+\frac{1}{4} u_{t}{ }^{2}, \\
T_{2}^{y}= & \frac{1}{3} \alpha u_{t} u_{x}{ }^{2}-\frac{1}{3} \alpha u u_{t x} u_{x}+\frac{1}{4} \beta u_{t} u_{x}+\frac{1}{8} v u_{t x x} u_{x}-\frac{1}{8} v u u_{t x x x}+\frac{1}{8} v u_{t} u_{x x x}-\frac{1}{8} v u_{t x} u_{x x} \\
& -\frac{1}{4} \beta u u_{t x} ;
\end{aligned}
$$

Case 3. For $\mathcal{Q}_{3}=u_{x}$, we have the conserved vector

$$
\begin{aligned}
T_{3}^{t}= & \frac{1}{4} u_{x}^{2}-\frac{1}{4} u u_{x x} \\
T_{3}^{x}= & \frac{2}{3} \alpha u_{x}^{2} u_{y}+\frac{1}{3} \alpha u u_{x} u_{x y}+\frac{5}{8} v u_{x} u_{x x y}+\frac{1}{4} \beta u_{x} u_{y}+\frac{1}{4} u_{t} u_{x}+\frac{1}{8} v u u_{x x x y}-\frac{3}{8} v u_{x x} u_{x y} \\
& +\frac{1}{8} v u_{x x x} u_{y}+\frac{1}{4} \beta u u_{x y}+\frac{1}{4} u u_{t x}, \\
T_{3}^{y}= & \frac{1}{3} \alpha u_{x}^{3}+\frac{1}{4} \beta u_{x}^{2}-\frac{1}{3} \alpha u u_{x} u_{x x}+\frac{1}{4} v u_{x} u_{x x x}-\frac{1}{8} v u u_{x x x x}-\frac{1}{4} \beta u u_{x x}-\frac{1}{8} v u_{x x}{ }^{2}
\end{aligned}
$$

Case 4 . For $\mathcal{Q}_{4}=u_{y}$, we have the conserved vector

$$
\begin{aligned}
T_{4}^{t}= & \frac{1}{4} u_{x} u_{y}-\frac{1}{4} u u_{x y} \\
T_{4}^{x}= & \frac{2}{3} \alpha u_{x} u_{y}^{2}+\frac{1}{4} \beta u_{y}^{2}-\frac{1}{3} \alpha u u_{x y} u_{y}+\frac{1}{2} v u_{x x y} u_{y}+\frac{1}{4} u_{t} u_{y}-\frac{3}{8} v u u_{x x y y}+\frac{1}{4} v u_{x} u_{x y y} \\
& -\frac{1}{8} v u_{x x} u_{y y}-\frac{1}{4} v u_{x y}{ }^{2}-\frac{1}{3} \alpha u u_{x} u_{y y}-\frac{1}{4} \beta u u_{y y}-\frac{1}{4} u u_{t y}, \\
T_{4}^{y}= & \frac{1}{3} \alpha u u_{x} u_{x y}+\frac{1}{3} \alpha u u_{x x} u_{y}+\frac{3}{8} v u u_{x x x y}+\frac{1}{4} \beta u u_{x y}+\frac{1}{2} u u_{t x}+\frac{1}{3} \alpha u_{x}{ }^{2} u_{y}+\frac{1}{8} v u_{x} u_{x x y} \\
& +\frac{1}{4} \beta u_{x} u_{y}-\frac{1}{8} v u_{x x} u_{x y}+\frac{1}{8} v u_{x x x} u_{y} ;
\end{aligned}
$$


Case 5. For $\mathcal{Q}_{5}=-2 \alpha t^{2} u_{t}-\alpha t x u_{x}-2 \alpha t y u_{y}-\alpha t u-\beta t x+x y$, we have the conserved vector

$$
\begin{aligned}
& T_{5}^{t}=-\frac{4}{3} \alpha^{2} t^{2} u u_{x} u_{x y}-\frac{2}{3} \alpha^{2} t^{2} u u_{y} u_{x x}-\alpha \beta t^{2} u u_{x y}-\alpha v t^{2} u u_{x x x y}-\frac{1}{2} \alpha t^{2} u u_{t x}-\frac{1}{2} \alpha t^{2} u_{t} u_{x} \\
& +\frac{1}{4} \alpha t x u u_{x x}+\frac{1}{2} \alpha t y u u_{x y}-\frac{1}{4} \alpha t x u_{x}^{2}-\frac{1}{2} \alpha t y u_{x} u_{y}+\frac{1}{4} \alpha t u u_{x}-\frac{1}{2} \beta t x u_{x}+\frac{1}{2} \beta t u \\
& +\frac{1}{2} x y u_{x}-\frac{1}{2} u_{y} \\
& T_{5}^{x}=\frac{1}{2} \alpha t^{2} u u_{t t}-\frac{1}{2} \beta^{2} t x u_{y}+\alpha t u u_{t}-\frac{1}{2} \beta t x u_{t}+\frac{1}{2} \beta v t u_{x y}-\frac{1}{4} \alpha x u u_{x}+\frac{3}{4} v x y u_{x x y} \\
& +\frac{1}{2} \beta x y u_{y}-\frac{1}{3} \alpha^{2} t_{x u u_{x}} u_{x y}+\frac{2}{3} \alpha^{2} \operatorname{tyuu}_{x} u_{y y}+\frac{2}{3} \alpha^{2} t y u u_{x y} u_{y}-\frac{1}{8} \alpha v t x u u_{x x x y} \\
& +\frac{3}{4} \alpha v t y u u_{x x y y}-\frac{1}{4} \alpha \beta t x u u_{x y}+\frac{1}{2} \alpha \beta t y u u_{y y}-\frac{5}{8} \alpha v t x u_{x} u_{x x y}-\frac{1}{2} \alpha v t y u_{x} u_{x y y} \\
& -\frac{5}{4} \alpha \beta t x u_{x} u_{y}+\frac{3}{8} \alpha v t x u_{x x} u_{x y}+\frac{1}{4} \alpha v t y u_{x x} u_{y y}-\frac{1}{8} \alpha v t x u_{x x x} u_{y}-\alpha v t y u_{x x y} u_{y} \\
& -\frac{4}{3} \alpha^{2} t^{2} u_{t} u_{x} u_{y}+\frac{3}{4} \alpha t^{2} v u u_{t x x y}+\frac{1}{2} \alpha t^{2} v u_{t x} u_{x y}+\frac{1}{4} \alpha t^{2} v u_{t y} u_{x x}-\frac{1}{4} \alpha t^{2} v u_{t x x} u_{y} \\
& -\frac{1}{2} \alpha t^{2} v u_{t x y} u_{x}-\frac{3}{4} \alpha t^{2} v u_{x x y} u_{t}+\frac{1}{2} \alpha \beta t^{2} u u_{t y}-\frac{1}{2} \alpha \beta t^{2} u_{t} u_{y}-\frac{2}{3} \alpha^{2} t x u_{x}^{2} u_{y} \\
& -\frac{4}{3} \alpha^{2} t y u_{x} u_{y}{ }^{2}+\alpha^{2} t u u_{x} u_{y}+\frac{1}{2} \alpha v t y u_{x y}{ }^{2}-\frac{1}{2} \alpha \beta t y u_{y}{ }^{2}-\frac{1}{4} \alpha t x u u_{t x}+\frac{1}{2} \alpha t y u u_{t y} \\
& +\frac{3}{2} \alpha v t u u_{x x y}+\alpha \beta t u u_{y}-\frac{1}{4} \alpha t x u_{t} u_{x}-\frac{1}{2} \alpha t y u_{t} u_{y}-\frac{1}{2} \alpha v t u_{x} u_{x y}-\frac{3}{4} \beta v t x u_{x x y} \\
& +\alpha x y u_{x} u_{y}+\frac{2}{3} \alpha^{2} t^{2} u u_{t x} u_{y}+\frac{2}{3} \alpha^{2} t^{2} u u_{t y} u_{x}+\frac{1}{4} u^{2} \alpha+\frac{1}{2} v u_{x}-\frac{1}{2} \alpha t^{2} u_{t}^{2}-\frac{1}{4} v x u_{x x} \\
& -\frac{1}{2} v y u_{x y}+\frac{1}{2} u_{t} x y \text {, } \\
& T_{5}^{y}=\frac{1}{3} \alpha^{2} \operatorname{txuu}_{x} u_{x x}-\frac{2}{3} \alpha^{2} \operatorname{tyuu}_{x} u_{x y}-\frac{2}{3} \alpha^{2} \operatorname{tyu} u_{x x} u_{y}+\frac{1}{4} \alpha \beta \operatorname{txu} u_{x x}+\frac{1}{8} \alpha v t x u u_{x x x x} \\
& -\frac{3}{4} \alpha v \operatorname{tyu} u_{x x x y}-\frac{1}{2} \alpha \beta \operatorname{tyu} u_{x y}-\frac{1}{4} \alpha v t x u_{x} u_{x x x}-\frac{1}{4} \alpha v t y u_{x} u_{x x y}-\frac{1}{2} \alpha \beta t y u_{x} u_{y} \\
& +\frac{1}{4} \alpha v \operatorname{ty} u_{x x} u_{x y}-\frac{1}{4} \alpha v t y u_{x x x} u_{y}+\frac{2}{3} \alpha^{2} t^{2} u u_{t x} u_{x}-\frac{1}{4} \alpha v t^{2} u_{x} u_{t x x}-\frac{1}{2} \alpha \beta t^{2} u_{x} u_{t} \\
& +\frac{1}{4} \alpha t^{2} v u u_{t x x x}+\frac{1}{4} \alpha t^{2} v u_{x x} u_{t x}-\frac{1}{4} \alpha t^{2} v u_{x x x} u_{t}+\frac{1}{2} \alpha \beta u t^{2} u_{t x}-\frac{2}{3} \alpha^{2} t y u_{x}^{2} u_{y} \\
& -\frac{3}{4} \alpha \beta t x u_{x}^{2}+\frac{1}{8} \alpha v t x u_{x x}{ }^{2}-\alpha t y u u_{t x}+\frac{3}{4} \alpha \beta t u u_{x}+\frac{3}{8} \alpha v t u u_{x x x}-\frac{1}{8} \alpha v t u_{x} u_{x x} \\
& -\frac{1}{4} \beta v t x u_{x x x}-\frac{1}{4} y v u_{x x}-\frac{1}{2} \beta y u+\frac{1}{2} \beta^{2} t u-\frac{2}{3} \alpha^{2} t^{2} u_{t} u_{x}{ }^{2}+\frac{1}{2} \alpha x y u_{x}^{2}-\frac{1}{2} \alpha y u u_{x} \\
& +\frac{1}{2} y \beta x u_{x}+\frac{1}{4} y v u_{x x x} x-\frac{1}{3} \alpha^{2} t x u_{x}^{3}+\frac{1}{3} \alpha^{2} t u u_{x}^{2}-\frac{1}{2} \beta^{2} t x u_{x}+\frac{1}{4} \beta v t u_{x x} ;
\end{aligned}
$$


Case 6. For $\mathcal{Q}_{6}=2 \beta t u_{y}+2 y u_{y}+4 t u_{t}+x u_{x}+u$, we have the conserved vector

$$
\begin{aligned}
& T_{6}^{t}=\frac{8}{3} u u_{x} u_{x y} \alpha t+\frac{4}{3} u u_{x x} u_{y} \alpha t+2 u u_{x x x y} v t+\frac{3}{2} u u_{x y} \beta t+u u_{t x} t-\frac{1}{4} u u_{x x} x-\frac{1}{2} u u_{x y} y \\
& -\frac{1}{4} u u_{x}+\frac{1}{2} u_{x} u_{y} \beta t+u_{x} u_{t} t+\frac{1}{4} u_{x}^{2} x+\frac{1}{2} u_{x} u_{y} y, \\
& T_{6}^{x}=\frac{1}{2} \beta y u_{y}{ }^{2}+\frac{1}{4} u_{t} u_{x} x+\frac{1}{2} u_{t} u_{y} y+\frac{1}{2} \beta^{2} t u_{y}{ }^{2}-u u_{t t} t-\frac{1}{2} v y u_{x y}{ }^{2}-\frac{3}{2} v u u_{x x y}+\frac{1}{2} v u_{x} u_{x y} \\
& +\frac{1}{4} x u u_{t x}-\frac{1}{2} y u u_{t y}-\beta u u_{y}+u_{t}^{2} t-u u_{t}+\frac{4}{3} \alpha \beta t u_{x} u_{y}^{2}-\frac{4}{3} \alpha t u u_{t x} u_{y}-\frac{4}{3} \alpha t u u_{t y} u_{x} \\
& -\frac{3}{4} \beta v t u u_{x x y y}+\frac{8}{3} \alpha t u_{t} u_{x} u_{y}+\frac{1}{2} \beta v t u_{x} u_{x y y}-\frac{1}{4} \beta v t u_{x x} u_{y y}+\beta v t u_{x x y} u_{y}+\frac{1}{3} \alpha x u u_{x} u_{x y} \\
& -\frac{2}{3} \alpha y u u_{x} u_{y y}-\frac{2}{3} \alpha y u u_{x y} u_{y}-\frac{2}{3} \alpha \beta t u u_{x} u_{y y}-\frac{2}{3} \alpha \beta t u u_{x y} u_{y}+\frac{2}{3} \alpha x u_{x}^{2} u_{y}+\frac{4}{3} \alpha y u_{x} u_{y}^{2} \\
& +\frac{1}{4} \beta x u_{x} u_{y}-\frac{1}{2} \beta^{2} t u u_{y y}-\frac{1}{2} \beta v t u_{x y}^{2}-\frac{3}{2} v t u u_{t x x y}-\frac{3}{2} \beta t u u_{t y}+\frac{3}{2} v t u_{t} u_{x x y}+\frac{3}{2} \beta t u_{t} u_{y} \\
& -v t u_{t x} u_{x y}+\frac{1}{2} v t u_{t x x} u_{y}+v t u_{t x y} u_{x}-\frac{1}{2} v t u_{t y} u_{x x}+\frac{1}{8} v x u u_{x x x y}-\frac{3}{4} v y u u_{x x y y} \\
& +\frac{5}{8} v x u_{x} u_{x x y}+\frac{1}{2} v y u_{x} u_{x y y}-\frac{3}{8} v x u_{x x} u_{x y}-\frac{1}{4} v y u_{x x} u_{y y}+\frac{1}{8} v x u_{x x x} u_{y}+v y u_{x x y} u_{y} \\
& -\alpha u u_{x} u_{y}+\frac{1}{4} \beta x u u_{x y}-\frac{1}{2} \beta y u u_{y y} \\
& T_{6}^{y}=\frac{2}{3} \alpha y u u_{y} u_{x x}+\frac{2}{3} \alpha \beta t u_{x}^{2} u_{y}-\frac{4}{3} \alpha t u u_{t x} u_{x}-\frac{1}{3} \alpha x u u_{x} u_{x x}+\frac{2}{3} \alpha y u u_{x} u_{x y}+\frac{1}{4} \beta \nu t u_{x} u_{x x y} \\
& +\frac{3}{4} \beta v t u u_{x x x y}-\frac{1}{4} \beta v t u_{x x} u_{x y}+\frac{1}{4} \beta v t u_{x x x} u_{y}+\frac{2}{3} \alpha \beta t u u_{y} u_{x x}+\frac{2}{3} \alpha \beta t u u_{x} u_{x y} \\
& +\frac{1}{4} v y u_{x x x} u_{y}+\frac{1}{2} u \beta^{2} t u_{x y}-\frac{1}{4} u \beta u_{x x} x+\frac{1}{2} u \beta u_{x y} y+\frac{4}{3} \alpha t u_{t} u_{x}{ }^{2}+\frac{2}{3} \alpha y u_{x}{ }^{2} u_{y} \\
& +\frac{1}{2} \beta^{2} t u_{x} u_{y}+\beta t u_{t} u_{x}+\frac{1}{2} v t u_{t x x} u_{x}+\frac{1}{4} v x u_{x} u_{x x x}+\frac{1}{4} v y u_{x} u_{x x y}+\frac{1}{2} \beta y u_{x} u_{y} \\
& -\frac{1}{2} v t u u_{t x x x}-\frac{1}{8} v x u u_{x x x x}+\frac{3}{4} v y u u_{x x x y}+\frac{1}{2} v t u_{t} u_{x x x}-\frac{1}{2} v t u_{t x} u_{x x}-\frac{1}{4} v y u_{x x} u_{x y} \\
& +u y u_{t x}-\frac{1}{3} \alpha u u_{x}^{2}+\frac{1}{4} \beta x u_{x}^{2}-\frac{1}{4} \beta u u_{x}+\frac{1}{8} v u_{x} u_{x x}-\frac{1}{8} v x u_{x x}{ }^{2}-\frac{3}{8} v u u_{x x x} \\
& +\frac{1}{3} \alpha x u_{x}^{3}
\end{aligned}
$$

Case 7. For $\mathcal{Q}_{7}=y F^{\prime}(t)-\alpha u_{x} F(t)$, we have the conserved vector

$$
\begin{aligned}
T_{7}^{t}= & -\frac{1}{2} y u_{x} F^{\prime}(t)+\left(\frac{1}{4} \alpha u u_{x x}-\frac{1}{4} \alpha u_{x}^{2}\right) F(t), \\
T_{7}^{x}= & -\frac{1}{2} y u F^{\prime \prime}(t)+\left(\alpha y u_{x} u_{y}-\frac{1}{4} \alpha u u_{x}+\frac{3}{4} v y u_{x x y}+\frac{1}{2} \beta y u_{y}-\frac{1}{2} u \beta+\frac{1}{2} u_{t} y-\frac{1}{4} v u_{x x}\right) F^{\prime}(t) \\
& -\left(\frac{1}{3} \alpha^{2} u u_{x} u_{x y}+\frac{2}{3} \alpha^{2} u_{x}^{2} u_{y}+\frac{5}{8} \alpha v u_{x} u_{x x y}+\frac{1}{4} \alpha \beta u_{x} u_{y}+\frac{1}{4} \alpha u_{t} u_{x}+\frac{1}{4} \alpha \beta u u_{x y}\right. \\
& \left.+\frac{1}{8} \alpha v u u_{x x x y}+\frac{1}{4} \alpha u u_{t x}-\frac{3}{8} \alpha v u_{x x} u_{x y}+\frac{1}{8} \alpha v u_{x x x} u_{y}\right) F(t) \\
T_{7}^{y}= & \left(\frac{1}{2} \alpha y u_{x}^{2}+\frac{1}{2} \beta y u_{x}+\frac{1}{4} v y u_{x x x x}\right) F^{\prime}(t)+\left(\frac{1}{3} \alpha^{3} u u_{x} u_{x x}-\frac{1}{3} \alpha^{2} u_{x}^{3}-\frac{1}{4} \alpha \beta u_{x}^{2}+\frac{1}{8} \alpha v u_{x x}^{2}\right. \\
& \left.-\frac{1}{4} \alpha v u_{x} u_{x x x}+\frac{1}{8} \alpha v u u_{x x x x}+\frac{1}{4} \alpha \beta u u_{x x}\right) F(t) ;
\end{aligned}
$$


Case 8. For $\mathcal{Q}_{8}=G(t)$, we have the conserved vector

$$
\begin{aligned}
T_{8}^{t} & =\frac{1}{2} u_{x} G(t), \\
T_{8}^{x} & =-\frac{1}{2} u G^{\prime}(t)+\left(\alpha u_{x} u_{y}+\frac{1}{2} \beta u_{y}+\frac{3}{4} v u_{x x y}+\frac{1}{2} u_{t}\right) G(t), \\
T_{8}^{y} & =\left(\frac{1}{2} \alpha u_{x}^{2}+\frac{1}{2} \beta u_{x}+\frac{1}{2} v u_{x x x}\right) G(t) .
\end{aligned}
$$

\subsection{Conservation Laws Utilizing Noether's Theorem}

We now construct conservation laws for Equation (1). We utilize the classical Noether's theorem $[34,43]$ to achieve this task.

Equation (1) has a second-order Lagrangian

$$
\mathcal{L}=\frac{1}{2}\left(v u_{x x} u_{x y}-u_{t} u_{x}-\alpha u_{x}^{2} u_{y}-\beta u_{x} u_{y}\right),
$$

since $\delta \mathcal{L} / \delta u=0$ on (1). Here, $\delta / \delta u$ is the Euler operator. Thus, we can determine the Noether symmetries of (1) by employing the Lagrangian (37) on the determining equation

$$
: X^{[2]} \mathcal{L}+\mathcal{L}\left\{D_{t}(\tau)+D_{x}(\xi)+D_{y}(\phi)\right\}-D_{t}\left(G^{t}\right)-D_{x}\left(G^{x}\right)-D_{y}\left(G^{y}\right)=0,
$$

with gauge functions $G^{t}, G^{x}$, and $G^{y}$. Expanding (38) and solving the resultant linear PDEs yield the following Noether symmetries and gauge functions:

$$
\begin{aligned}
& X_{1}=\frac{\partial}{\partial t}, G^{t}=0, G^{x}=0, G^{y}=0, \\
& X_{2}=\frac{\partial}{\partial y}, G^{t}=0, G^{x}=0, G^{y}=0, \\
& X_{3}=g(t) \frac{\partial}{\partial u}, G^{t}=0, G^{x}=-\frac{1}{2} u g^{\prime}(t), G^{y}=0, \\
& X_{4}=2 \alpha t \frac{\partial}{\partial y}+x \frac{\partial}{\partial u}, G^{t}=-\frac{1}{2} u, G^{x}=0, G^{y}=-\frac{1}{2} \beta u, \\
& X_{5}=\alpha f(t) \frac{\partial}{\partial x}+y f^{\prime}(t) \frac{\partial}{\partial u}, G^{t}=0, G^{x}=-\frac{1}{2} u y f^{\prime \prime}(t)-\frac{1}{2} \beta u f^{\prime}(t), G^{y}=0, \\
& X_{6}=4 \alpha t \frac{\partial}{\partial t}+\alpha x \frac{\partial}{\partial x}+2 \alpha y \frac{\partial}{\partial y}-(\beta x+\alpha u) \frac{\partial}{\partial u}, G^{t}=\frac{1}{2} \beta u, G^{x}=0, G^{y}=\frac{1}{2} \beta^{2} u .
\end{aligned}
$$

Thus, the conserved vectors corresponding to the above obtained Noether point symmetries, using [41]

$$
T^{k}=\mathcal{L} \xi^{k}+\left(\eta^{\alpha}-u_{x^{j}}^{\alpha} \xi^{j}\right)\left(\frac{\partial \mathcal{L}}{\partial u_{x^{k}}^{\alpha}}-\sum_{l=1}^{k} D_{x^{l}}\left(\frac{\partial \mathcal{L}}{\partial u_{x^{l} x^{k}}^{\alpha}}\right)\right)+\sum_{l=k}^{n}\left(\zeta_{l}^{\alpha}-u_{x^{l} x^{j}}^{\alpha} \xi^{j}\right) \frac{\partial \mathcal{L}}{\partial u_{x^{k} x^{l}}^{\alpha}}
$$

are given by, respectively,

$$
\begin{aligned}
& T_{1}^{t}=-\frac{1}{2} \alpha u_{x}^{2} u_{y}-\frac{1}{2} \beta u_{x} u_{y}+\frac{1}{2} v u_{x x} u_{x y} \\
& T_{1}^{x}=\alpha u_{t} u_{x} u_{y}+\frac{3}{4} v u_{t} u_{x x y}-\frac{1}{4} v u_{x x} u_{t y}-\frac{1}{2} v u_{t x} u_{x y}+\frac{1}{2} \beta u_{t} u_{y}+\frac{1}{2} u_{t}^{2} \\
& T_{1}^{y}=\frac{1}{2} \alpha u_{t} u_{x}^{2}+\frac{1}{2} \beta u_{t} u_{x}+\frac{1}{4} v u_{t} u_{x x x}-\frac{1}{4} v u_{x x} u_{t x}
\end{aligned}
$$




$$
\begin{aligned}
& T_{2}^{t}=\frac{1}{2} u_{x} u_{y} \\
& T_{2}^{x}=\frac{1}{2} u_{t} u_{y}+\alpha u_{x} u_{y}^{2}+\frac{3}{4} v u_{y} u_{x x y}-\frac{1}{2} v u_{x y}^{2}-\frac{1}{4} v u_{x x} u_{y y}+\frac{1}{2} \beta u_{y}^{2}, \\
& T_{2}^{y}=\frac{1}{4} v u_{x x} u_{x y}+\frac{1}{4} v u_{x x x} u_{y}-\frac{1}{2} u_{t} u_{x} ; \\
& T_{3}^{t}=-\frac{1}{2} u_{x} g(t) \\
& T_{3}^{x}=\left(-\alpha u_{x} u_{y}-\frac{3}{4} v u_{x x y}-\frac{1}{2} \beta u_{y}-\frac{1}{2} u_{t}\right) g(t)+\frac{1}{2} u g^{\prime}(t), \\
& T_{3}^{y}=\left(-\frac{1}{2} \alpha u_{x}^{2}-\frac{1}{2} \beta u_{x}-\frac{1}{4} v u_{x x x}\right) g(t) ; \\
& T_{4}^{t}=\frac{1}{2} u-\frac{1}{2} x u_{x}+\alpha t u_{x} u_{y} \\
& T_{4}^{x}=\frac{1}{2} v u_{x y}-\frac{1}{2} x u_{t}-\alpha x u_{x} u_{y}-\frac{1}{2} \beta x u_{y}-\frac{1}{2} v x u_{x x y}+\alpha t u_{t} u_{y}+2 \alpha^{2} t u_{x} u_{y}^{2}+\alpha v t u_{y} u_{x x y} \\
& -\alpha v t u_{x y}^{2}-\alpha v t u_{x x} u_{y y}+\alpha \beta t u_{y}^{2} \\
& T_{4}^{y}=\frac{1}{2} \beta u+\alpha v t u_{y} u_{x x x}-\frac{1}{2} v x u_{x x x}-\frac{1}{2} \beta x u_{x}-\frac{1}{2} \alpha x u_{x}^{2}-\alpha t u_{t} u_{x}+\alpha v t u_{x x} u_{x y} ; \\
& T_{5}^{t}=\frac{1}{2} \alpha u_{x}^{2} f(t)-\frac{1}{2} y u_{x} f^{\prime}(t) \\
& T_{5}^{x}=\left(\frac{1}{2} \alpha^{2} u_{x}^{2} u_{y}+\frac{3}{4} \alpha v u_{x} u_{x x y}-\frac{1}{4} \alpha v u_{x x} u_{x y}\right) f(t)+\left(-\frac{1}{2} y u_{t}-\alpha y u_{x} u_{y}-\frac{3}{4} v y u_{x x y}\right. \\
& \left.+\frac{1}{4} v u_{x x}-\frac{1}{2} \beta y u_{y}+\frac{1}{2} \beta u\right) f^{\prime}(t)+\frac{1}{2} y u f^{\prime \prime}(t), \\
& T_{5}^{y}=\left(\frac{1}{2} \alpha^{2} u_{x}^{3}+\frac{1}{2} \alpha \beta u_{x}^{2}+\frac{1}{4} \alpha v u_{x x x} u_{x}-\frac{1}{4} \alpha v u_{x x}^{2}\right) f(t)-\left(\frac{1}{2} \alpha y u_{x}^{2}+\frac{1}{2} \beta y u_{x}\right. \\
& \left.+\frac{1}{4} v y u_{x x x}\right) f^{\prime}(t) \\
& T_{6}^{t}=\frac{1}{2} x \alpha u_{x}^{2}-2 t \alpha^{2} u_{y} u_{x}^{2}+\frac{1}{2} x \beta u_{x}+\frac{1}{2} \alpha u u_{x}+y \alpha u_{y} u_{x}-2 t \alpha \beta u_{y} u_{x}+2 t \alpha v u_{x y} u_{x x} \\
& -\frac{1}{2} \beta u \\
& T_{6}^{x}=\frac{1}{2} x u_{y} u_{x}^{2} \alpha^{2}+2 y u_{y}^{2} u_{x} \alpha^{2}+u u_{y} u_{x} \alpha^{2}+4 t u_{y} u_{x} u_{t} \alpha^{2}+y \beta u_{y}^{2} \alpha-y v u_{x y}^{2} \alpha+2 t u_{t}^{2} \alpha \\
& +\frac{1}{2} \beta u u_{y} \alpha+x \beta u_{y} u_{x} \alpha-v u_{x} u_{x y} \alpha-\frac{3}{4} v u_{y} u_{x x} \alpha-\frac{1}{2} y v u_{y y} u_{x x} \alpha-\frac{1}{4} x v u_{x y} u_{x x} \alpha \\
& +\frac{3}{4} v u u_{x x y} \alpha+\frac{3}{2} y v u_{y} u_{x x y} \alpha+\frac{3}{4} x v u_{x} u_{x x y} \alpha+\frac{1}{2} u u_{t} \alpha+y u_{y} u_{t} \alpha+2 t \beta u_{y} u_{t} \alpha \\
& +3 t v u_{x x y} u_{t} \alpha-t v u_{x x} u_{t y} \alpha-2 t v u_{x y} u_{t x} \alpha+\frac{1}{2} x \beta^{2} u_{y}-\frac{1}{2} \beta v u_{x y}+\frac{3}{4} x \beta v u_{x x y} \\
& +\frac{1}{2} x \beta u_{t}
\end{aligned}
$$




$$
\begin{aligned}
T_{6}^{y}= & \frac{1}{2} x \alpha^{2} u_{x}^{3}+x \alpha \beta u_{x}^{2}+\frac{1}{2} \alpha^{2} u u_{x}^{2}+2 t \alpha^{2} u_{t} u_{x}^{2}+\frac{1}{2} x \beta^{2} u_{x}+\frac{1}{2} \alpha \beta u u_{x}-\frac{1}{2} \alpha v u_{x x} u_{x} \\
& +\frac{1}{4} x \alpha v u_{x x x} u_{x}-y \alpha u_{t} u_{x}+2 t \alpha \beta u_{t} u_{x}-\frac{1}{4} x \alpha v u_{x x}^{2}-\frac{1}{4} \beta v u_{x x}+\frac{1}{2} y \alpha v u_{x y} u_{x x} \\
& +\frac{1}{4} x \beta v u_{x x x}+\frac{1}{4} \alpha v u u_{x x x}+\frac{1}{2} y \alpha v u_{y} u_{x x x}+t \alpha v u_{x x x} u_{t}-t \alpha v u_{x x} u_{t x}-\frac{1}{2} \beta^{2} u
\end{aligned}
$$

It should be noted that we derived eight conservation laws of (1) by invoking the multiplier method whereas Noether's theorem yielded six conservation laws. However, we see that only one conservation law is common when using both methods. The conservation laws obtained with the Lagrange multiplier method could be obtained by means of Noether's theorem using contact transformations. Thus, we conclude that there are benefits and constraints of the different methods used when deriving the conservation laws of nonlinear partial differential equations.

\section{Concluding Remarks}

In this paper, we investigated the generalized two-dimensional generalized shallow water wave Equation (1). We determined the Lie point symmetries of this equation, performed symmetry reductions, and reduced it to an NLODE (8). This NLODE (8) was integrated directly and its solution was obtained in the form of an incomplete elliptic integral. Moreover, Kudrayshov's method was employed to obtain the solution of the NLODE (8). These solutions were presented graphically. Finally, we used the multiplier method and Noether's theorem to derive the conservation laws of (1). The multiplier method yielded eight multipliers, which gave eight local conservation laws for Equation (1), whereas Noether's theorem provided us with six local conservation laws, which included energy and linear momentum conservation laws.

Author Contributions: Conceptualization, C.M.K. and K.P.; Methodology, C.M.K.; Software, K.P.; Validation, C.M.K.; Writing—original draft, K.P.; Writing—review \& editing, C.M.K. All authors have read and agreed to the published version of the manuscript.

Funding: This research received no external funding.

Institutional Review Board Statement: Not applicable.

Informed Consent Statement: Not applicable.

Data Availability Statement: Not applicable.

Acknowledgments: The authors thank OD Adeyemo for fruitful discussions and North-West University, Mafikeng campus, for their continued support. Additionally, the authors thank the reviewers for their positive suggestions, which helped to improve the paper enormously.

Conflicts of Interest: The authors declare no conflict of interest.

\section{References}

1. Du, X.X.; Tian, B.; Qu, Q.X.; Yuan, Y.Q.; Zhao, X.H. Lie group analysis, solitons, self-adjointness and conservation laws of the modified Zakharov-Kuznetsov equation in an electron-positron-ion magnetoplasma. Chaos Solitons Fractals 2020, $134,109709$. [CrossRef]

2. Gao, X.Y. Mathematical view with observational/experimental consideration on certain $(2+1)$-dimensional waves in the cosmic/laboratory dusty plasmas. Appl. Math. Lett. 2019, 91, 165-172. [CrossRef]

3. Zhang, C.R.; Tian, B.; Qu, Q.X.; Liu, L.; Tian, H.Y. Vector bright solitons and their interactions of the couple Fokas-Lenells system in a birefringent optical fiber, Z. Angew. Math. Phys. 2020, 71, 1-19.

4. Gao, X.Y.; Guo, Y.J.; Shan, W.R. Water-wave symbolic computation for the Earth, Enceladus and Titan: The higher-order BoussinesqBurgers system, auto-and non-auto-Bäcklund transformations. Appl. Math. Lett. 2020, 104, 106170. [CrossRef]

5. Benoudina, N.; Zhang, Y.; Khalique, C.M. Lie symmetry analysis, optimal system, new solitary wave solutions and conservation laws of the Pavlov equation. Commun. Nonlinear Sci. Numer. Simul. 2021, 94, 105560. [CrossRef]

6. Gandarias, M.L.; Duran, M.R.; Khalique, C.M. Conservation laws and travelling wave solutions for double dispersion equations in $(1+1)$ and $(2+1)$ dimensions. Symmetry 2020, 12, 950. [CrossRef] 
7. Shafiq, A.; Khalique, C.M. Lie group analysis of upper convected Maxwell fluid flow along stretching surface. Alex. Eng. J. 2020, 59, 2533-2541. [CrossRef]

8. Rosa, M.; Chuliána, S.; Gandarias, M.L.; Traciná, R. Application of Lie point symmetries to the resolution of an interface problem in a generalized Fisher equation. Physica D 2020, 405, 132411. [CrossRef]

9. Wazwaz, A.M. Exact soliton and kink solutions for new $(3+1)$-dimensional nonlinear modified equations of wave propagation. Open Eng. 2017, 7, 169-174. [CrossRef]

10. Wazwaz, A.M.; El-Tantawy, S. A new (3+1)-dimensional generalized Kadomtsev-Petviashvili equation. Nonlinear Dyn. 2016, 84, 1107-1112. [CrossRef]

11. Wazwaz, A.M. Multiple-soliton solutions for a $(3+1)$-dimensional generalized KP equation. Commun. Nonlinear. Sci. Numer. Simul. 2012, 17, 491-495. [CrossRef]

12. Wazwaz, A.M. The tanh and sine-cosine method for compact and noncompact solutions of nonlinear Klein-Gordon equation. Appl. Math. Comput. 2005, 167, 1179-1195. [CrossRef]

13. Yang, J.Y.; Ma, W.X.; Khalique, C.M. Determining lump solutions for a combined soliton equation in (2+1)-dimensions. Eur. Phys. J. Plus 2020, 135, 494. [CrossRef]

14. Yildirim, Y.; Yasar, E. An extended Korteweg-de Vries equation: Multi-soliton solutions and conservation laws. Nonlinear Dyn. 2017, 90, 1571-1579. [CrossRef]

15. Mhlanga, I.E.; Khalique, C.M. A study of a generalized Benney-Luke equation with time-dependent coefficients. Nonlinear Dyn. 2017, 90, 1535-1544. [CrossRef]

16. Ablowitz, M.J.; Clarkson, P.A. Solitons, Nonlinear Evolution Equations and Inverse Scattering; Cambridge University Press: Cambridge, UK, 1991.

17. Zhang, L.; Khalique, C.M. Classification and bifurcation of a class of second-order ODEs and its application to nonlinear PDEs. Discret. Contin. Dyn.-Syst. 2018, 11,777-790. [CrossRef]

18. Kudryashov, N.A. Simplest equation method to look for exact solutions of nonlinear differential equations. Chaos Solitons Fractals 2005, 24, 1217-1231. [CrossRef]

19. Kudryashov, N.A.; Loguinova, N.B. Extended simplest equation method for nonlinear differential equations. Appl. Math. Comput. 2008, 205, 396-402 [CrossRef]

20. Kudryashov, N.A. One method for finding exact solutions of nonlinear differential equations. Commun. Nonlinear Sci. Numer. Simul. 2012, 17, 2248-2253 [CrossRef]

21. Hirota, R. The Direct Method in Soliton Theory; Cambridge University Press: Cambridge, UK, 2004.

22. Gu, C.H. Soliton Theory and Its Application; Zhejiang Science and Technology Press: Hangzhou, China, 1990.

23. Matveev, V.B.; Salle, M.A. Darboux Transformations and Solitons; Springer: New York, NY, USA, 1991.

24. Wang, M.; Zhou, Y.; Li, Z. Application of a homogeneous balance method to exact solutions of nonlinear equations in mathematical physics. Phys. Lett. A 1996, 216, 67-75. [CrossRef]

25. Wang, M.; Li, X.; Zhang, J. The $\left(G^{\prime} / G\right)$-Expansion method and travelling wave solutions for linear evolution equations in mathematical physics. Phys. Lett. A 2005, 24, 1257-1268.

26. Olver, P.J. Applications of Lie Groups to Differential Equations, 2nd ed.; Springer: Berlin/Heidelberg, Germany, 1993.

27. Ibragimov, N.H. CRC Handbook of Lie Group Analysis of Differential Equations; CRC Press: Boca Raton, FL, USA, $1994 ;$ Volume 1.

28. Ibragimov, N.H. Elementary Lie Group Analysis and Ordinary Differential Equations; John Wiley \& Sons: Chichester, NY, USA, 1999.

29. Khalique, C.M.; Adem, K.R. Explicit solutions and conservation laws of a (2+1)-dimensional KP-Joseph-Egri equation with power law nonlinearity. J. Appl. Nonlinear Dyn. 2018, 7, 1-9. [CrossRef]

30. Khalique, C.M.; Adeyemo, O.D.I. Simbanefayi, On optimal system, exact solutions and conservation laws of the modified equal-width equation. J. Appl. Math. Nonlinear Sci. 2018, 3, 409-418. [CrossRef]

31. Khalique, C.M.; Moleleki, L.D. A (3+1)-dimensional generalized BKP-Boussinesq equation: Lie group approach. Results Phys. 2019, 13, 102239. [CrossRef]

32. Khalique, C.M.; Plaatjie, K.; Simbanefayi, I. Exact solutions of equal-width equation and its conservation laws. Open Phys. 2019, 17, 505-511. [CrossRef]

33. Motsepa, T.; Khalique, C.M.; Gandarias, M.L. Symmetry analysis and conservation laws of the Zoomeron equation. Symmetry 2017, 9, 27. [CrossRef]

34. Noether, E. Invariante variationsprobleme, Nachr. v. d. Ges. d. Wiss. zu Göttingen. Math.-Phys. Klasse 1918, 2, $235-257$.

35. Bluman, G.W.; Cheviakov, A.F.; Anco, S.C. Applications of Symmetry Methods to Partial Differential Equations; Springer: New York, NY, USA, 2010.

36. Leveque, R.J. Numerical Methods for Conservation Laws, 2nd ed.; Birkhäuser-Verlag: Basel, Switzerland, 1992.

37. Ibragimov, N.H. A new conservation theorem. J. Math. Anal. Appl. 2007, 333, 311-328. [CrossRef]

38. Naz, R.; Mahomed, F.M.; Mason, D.P. Comparison of different approaches to conservation laws for some partial differential equations in fluid mechanics. Appl. Math. Comput. 2008, 205, 212-230. [CrossRef]

39. Sjöberg, A. On double reductions from symmetries and conservation laws. Nonlinear Anal. Real World Appl. 2009, 10, 3472-3477. [CrossRef]

40. Yasar, E.; Özer, T. On symmetries, conservations laws and similarity solutions of foam drainage equation, Internat. J. Non-Linear Mech. 2011, 46, 357-362. [CrossRef] 
41. Sarlet, W. Comment on 'Conservation laws of higher order nonlinear PDEs and the variational conservation laws in the class with mixed derivatives'. J. Phys. A Math. Theor. 2010, 43, 458001. [CrossRef]

42. Motsepa, T.; Abudiab, M.; Khalique, C.M. A Study of an extended generalized $(2+1)$-dimensional Jaulent-Miodek equation. Int. J. Nonlin. Sci. Num. 2018, 19, 391-395. [CrossRef]

43. Anco, S.C. Generalization of Noether's theorem in modern form to non-variational partial differential equations. In Recent Progress and Modern Challenges in Applied Mathematics, Modeling and Computational Science; Fields Institute Communications; Springer: New York, NY, USA, 2017; Volume 79, pp. 119-182.

44. Khalique, C.M.; Abdallah, S.A. Coupled Burgers equations governing polydispersive sedimentation; a Lie symmetry approach. Results Phys. 2020, 16, 102967. [CrossRef]

45. Bruzón, M.S.; Gandarias, M.L. Traveling wave solutions of the $\mathrm{K}(\mathrm{m}, \mathrm{n})$ equation with generalized evolution. Math. Meth. Appl. Sci. 2018, 41, 5851-5857. [CrossRef]

46. Xin, X.; Zhang, L.; Xia, Y.; Liu, H. Nonlocal symmetries and exact solutions of the $(2+1)$-dimensional generalized variable coefficient shallow water wave equation. Appl. Math. Lett. 2019, 94, 112-119. [CrossRef]

47. Lan, Z.; Gao, Y.; Yang, J.; Su, C.; Zuo, D. Solitons, bäcklund transformation, lax pair, and infinitely many conservation law for a $(2+1)$-dimensional generalised variable-coefficient shallow water wave equation. Naturforsch 2016, 71, 69-79. [CrossRef]

48. Yildirim, Y.; Yasar, E. A $(2+1)$ dimensional breaking soliton equation: Solutions and conservation laws, Chaos, Solitons and Fractals. Chaos Solitons Fractals 2018, 107, 146-155. [CrossRef]

49. Wazwaz, A.M. Integrable (2+1)-dimensional and $(3+1)$-dimensional breaking soliton equations. Phys. Scr. 2010, 81, 035005 . [CrossRef]

50. Wazwaz, A.M. A variety of completely integrable Calogero-Bogoyavlenskii-Schiff equations with time-dependent coefficients. Int. J. Numer. Method H 2020, doi:10.1108/hff-01-2020-0015/full/html. [CrossRef]

51. Wazwaz, A.M. Multiple-soliton solutions for the Calogero-Bogoyavlenskii-Schiff, Jimbo-Miwa and YTSF equations. Appl. Math. Comput. 2008, 203, 592-597. [CrossRef]

52. Wazwaz, A.M. New solutions of distinct physical structures to high-dimensional nonlinear evolution equations. Appl. Math. Comput. 2008, 196, 363-370. [CrossRef]

53. Salem, S.; Kassem, M.; Mabrouk, S.M. Similarity Solution of $(2+1)$-dimensional Calogero-Bogoyavlenskii-Schiff equation Lax pair. Am. J. Appl. Math. 2019, 7, 137-144. [CrossRef]

54. Shakeel, M.; Mohyud-Din, S.T. Improved $\left(G^{\prime} / G\right)$-Expansion and extended tanh methods for $(2+1)$-dimensional CalogeroBogoyavlenskii-Schiff equation. Alex. Eng. J. 2015, 54, 27-33. [CrossRef]

55. Najafi, M.; Najafi, M.; Arbabi, S. New application of $\left(G^{\prime} / G\right)$-Expansion method for generalized $(2+1)$-dimensional nonlinear evolution equations. J. Eng. Math. 2013, 5, 595-603. [CrossRef]

56. Darvishi, M.T.; Najafi, M.; Najafi, M. New application of EHTA for the generalized (2+1)-dimensional nonlinear evolution equations. Int. J. Math. Comput. Sci. 2010, 6, 132-138.

57. Najafi, M.; Najafi, M.; Arbabi, S. New exact solutions for the generalized ( $2+1)$-dimensional nonlinear evolution equations by tanh-coth method. Int. J. Modern Theor. Phys. 2013, 2, 79-85.

58. Najafi, M.; Arbabi, S.; Najafi, M. New application of sine-cosine method for the generalized $(2+1)$-dimensional nonlinear evolution equations. Int. J. Adv. Math. Sci. 2013, 1, 45-49. [CrossRef]

59. Aminakbari, N.; Gu, Y.; Yuan, W. Meromorphic exact solutions of the $(2+1)$-dimensional generalized Calogero-BogoyavlenskiiSchiff equation. Open Math. J. 2020, 18, 1342-1351. [CrossRef]

60. Abramowitz, M.; Stegun, I. Handbook of Mathematical Functions; Dover: New York, NY, USA, 1972.

61. Kudryashov, N.A. Analytical Theory of Nonlinear Differential Equations; Institute of Computer Investigations: Moskow, Russia, 2004.

62. Motsepa, T.; Khalique, C.M. Cnoidal and snoidal waves solutions and conservation laws of a generalized $(2+1)$-dimensional KdV equation. In Proceedings of the 14th Regional Conference on Mathematical Physics, Islamabad, Pakistan, 9-14 November 2015; World Scientific Publishing Co. Pte Ltd.: Singapore, 2018; pp. 253-263. 